\title{
EFFICIENT TRIGGERING WITH THE LHCb DETECTOR FOR PRECISE CP MEASUREMENTS*
}

\author{
N. TUNING for the LHCb Collaboration \\ CERN, CH-1211 Geneva 23, Switzerland \\ E-mail: Niels.Tuning@cern.ch
}

\begin{abstract}
The LHCb experiment is designed to study CP violation in B-decays at the LHC machine at a luminosity of $2 \cdot 10^{32} \mathrm{~cm}^{-2} \mathrm{~s}^{-1}$ and a rate of $40 \mathrm{MHz}$. It is crucial to efficiently select those pp-interactions containing the B-meson decays of interest. The expected physics reach of the LHCb experiment has recently been re-evaluated, after changes in the detector geometry and using full detector simulation and reconstruction. Both the trigger strategy and performance, as well as the new results on the expected physics reach will be presented for a few specific channels.
\end{abstract}

\section{Introduction}

The $\mathrm{LHCb}$ experiment is designed to study $\mathrm{CP}$ violation and rare decays in the B-system in pp-collisions at the LHC machine with a center-of-mass energy of $\sqrt{s}=14 \mathrm{TeV}$. With a $\mathrm{b} \overline{\mathrm{b}}$ cross section of $0.5 \mathrm{mb}$ and a nominal luminosity of $2 \cdot 10^{32} \mathrm{~cm}^{-2} \mathrm{~s}^{-1}$, a total amount of $10^{12} \mathrm{~b} \overline{\mathrm{b}}$-pairs will be produced per year. This will allow precise measurements of the angles $\beta, \gamma$ and $\chi$ of the well known unitarity triangles, see Fig. 1. The large production of $\mathrm{B}^{0}$ and $\mathrm{B}_{\mathrm{s}}^{0}$-mesons leads to a significant discovery potential for physics beyond the standard model ${ }^{1}$. New particles in loop diagrams might introduce new $\mathrm{CP}$ violating phases of the couplings, or alter the branching ratios of rare decays by changing the strength of the couplings.

The LHCb detector is a single arm spectrometer covering the range $1.9<\eta<4.9$ and has recently been redesigned (Sec. 2) in order to increase the trigger efficiency (Sec. 3) and to optimize the physics performance of the experiment (Sec. 4).

*Proceedings of a talk presented at the 8th ICATPP Conference on Advanced Technology and Particle physics, Como, Italy, October 6-11 2003. 


\section{The re-optimized $\mathrm{LHCb}$ detector}

The LHCb apparatus ${ }^{2}$ consists of a vertex locator (VELO), a tracking system with a dipole magnet, two Ring Imaging Cherenkov counters (RICH), electromagnetic and hadronic calorimeters, and a muon system. The VELO is made of 21 stations, consisting of two silicon disks each. The full silicon Trigger Tracker (TT) consists of four planes, split into two pairs that are 30 $\mathrm{cm}$ apart, and is placed in front of the magnet, inside the magnetic fringe field, see Fig. 1. The tracking system is made of three tracking stations (of four planes each) behind the magnet with silicon sensors in the inner region and straw tubes in the outer region. This setup ensures a high track finding efficiency of $95 \%$ and an excellent proper time resolution of $40 \mathrm{fs}$.
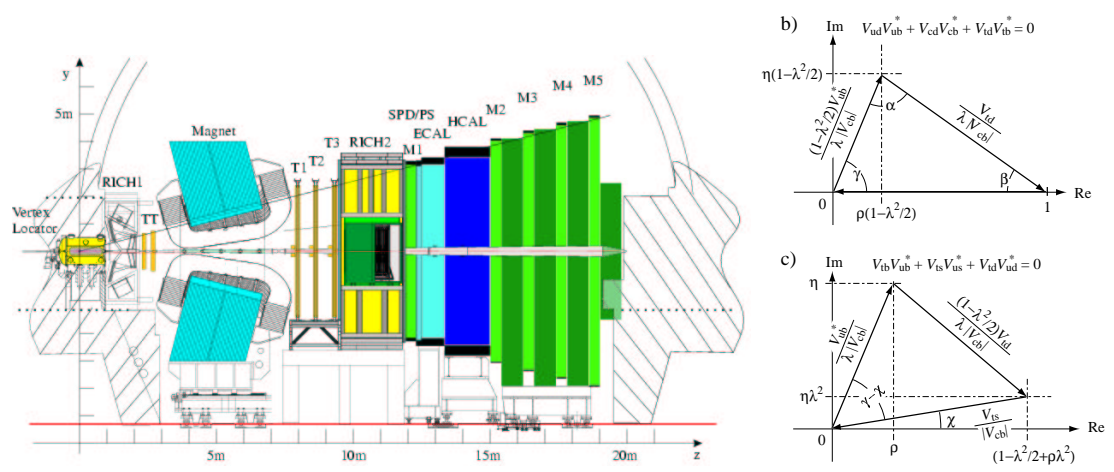

Figure 1. (a) The re-optimized LHCb detector. (b-c) Two unitarity triangles.

\section{Trigger}

The trigger has the challenging task to efficiently select interesting Bdecays, given the low branching ratios, the high interaction rate, the low b-cross section compared to the total cross section and the high-multiplicity environment. The reduction of the interaction rate of $40 \mathrm{MHz}$ to the rate of a few hundred $\mathrm{Hz}$ with which the events will be written to storage, is achieved in a three-level system ${ }^{3}$.

\subsection{Level-O}

The first trigger level (L0) uses the high mass of the B-mesons, resulting in decay products with large transverse momentum $\left(p_{\mathrm{T}}\right)$. High- $p_{\mathrm{T}}$ photons, 
electrons, hadrons and muons are reconstructed in the electromagnetic, hadronic calorimeter and the muon system, respectively. The pileup system, consisting of two VELO disks upstream the interaction region, identifies multiple interactions and is used to suppress events with multiple vertices or with large hit multiplicity. Hence more low-multiplicity events are retained, while keeping the same accept rate, and therefore both the average event size and computing time are reduced, with even a slight increased selection efficiency. The pileup information, together with the highest- $p_{\mathrm{T}}$ photon, electron and hadron, and the two highest- $p_{\mathrm{T}}$ muons, are passed to the L0 decision unit, which forms the final decision. The fully synchronous L0-trigger, with a fixed latency of $4 \mu \mathrm{s}$, is implemented in custom boards and reduces the rate to $1 \mathrm{MHz}$. Efficiencies of approximately $90 \%, 70 \%$ and $50 \%$ are achieved for events with muons, hadrons and photons respectively, as is shown in Table 1.

\subsection{Level-1}

The second trigger level (L1) reduces the rate further to $40 \mathrm{kHz}$, using information from the VELO and the TT station. The algorithm will be implemented on a commodity processors farm, that will be shared with the High Level Trigger (HLT) and the offline reconstruction. The available time at $\mathrm{L} 1$ is on average $\sim 1 \mathrm{~ms}^{\mathrm{a}}$, with a maximum latency of $52.4 \mathrm{~ms}$ given by the L1 buffer size. Large impact parameter tracks are reconstructed and matched to clusters in the TT station. The fringe field between the VELO

Table 1. The estimated trigger performance is listed for a few selected channels, together with the branching ratio of the B-decay.

\begin{tabular}{|l|c|c|c|}
\hline Channel & L0 eff. & L1 eff. & BR $\left(10^{-6}\right)$ \\
\hline \hline $\mathrm{B}^{0} \rightarrow \mathrm{J} / \psi\left(\mu^{+} \mu^{-}\right) \mathrm{K}_{\mathrm{S}}$ & $89.3 \pm 0.5 \%$ & $67.7 \pm 0.9 \%$ & $19.8 \pm 1.3$ \\
$\mathrm{~B}_{\mathrm{s}}^{0} \rightarrow \mathrm{J} / \psi\left(\mu^{+} \mu^{-}\right) \phi$ & $89.7 \pm 0.1 \%$ & $71.4 \pm 0.2 \%$ & $31 \pm 11$ \\
\hline $\mathrm{B}_{\mathrm{s}}^{0} \rightarrow \mathrm{D}_{\mathrm{S}}^{ \pm} \mathrm{K}^{\mp}$ & $47.2 \pm 0.3 \%$ & $62.6 \pm 0.4 \%$ & $10 \pm 4$ \\
$\mathrm{~B}^{0} \rightarrow \pi^{+} \pi^{-}$ & $53.6 \pm 0.4 \%$ & $62.7 \pm 0.5 \%$ & $4.8 \pm 0.5$ \\
$\mathrm{~B}_{\mathrm{s}}^{0} \rightarrow \mathrm{K}^{+} \mathrm{K}^{-}$ & $51.8 \pm 0.3 \%$ & $60.0 \pm 0.4 \%$ & $18.5 \pm 1.2$ \\
$\mathrm{~B}^{0} \rightarrow \mathrm{D}^{0} \mathrm{~K}^{* 0}$ & $53.0 \pm 1.4 \%$ & $66.7 \pm 1.8 \%$ & $1.2 \pm 0.3$ \\
$\mathrm{~B}_{\mathrm{s}}^{0} \rightarrow \mathrm{D}_{\mathrm{s}}^{-} \pi^{+}$ & $49.4 \pm 0.6 \%$ & $63.0 \pm 0.9 \%$ & $120 \pm 30$ \\
\hline $\mathrm{B}^{0} \rightarrow \mathrm{K}^{* 0} \gamma$ & $72.9 \pm 1.0 \%$ & $51.9 \pm 1.4 \%$ & $29 \pm 3$ \\
\hline
\end{tabular}

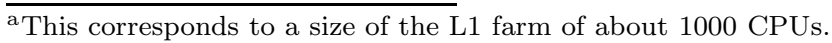


and the TT is sufficient to obtain momentum information with a precision of $\sim 30 \%$. In addition, the tracks are matched to muon candidates from L0, enhancing the performance for channels containing muons. Using two high impact parameter tracks with the highest $p_{\mathrm{T}}$, efficiencies between $50 \%$ and $70 \%$ are achieved, see Table 1.

\subsection{High Level Trigger}

The full detector information is available at the High Level Trigger (HLT). The tracking stations (T1-T3) provide more precise momentum information, with which the L1 decision can be confirmed within $4 \mathrm{~ms}$, reducing the rate by a factor 2 , while retaining signal efficiencies above $95 \%$. The remaining time $(\sim 14 \mathrm{~ms})$ is used to select the individual channels with an output rate of $\sim 10 \mathrm{~Hz}$ per channel.

\section{Physics Reach}

The current $e^{+} e^{-}$B-factories have measured CP violation in the B-system with very high accuracy ${ }^{4}, \sin 2 \beta=0.731 \pm 0.055$, and in excellent agreement with the indirect measurements of $\left|V_{u b}\right| /\left|V_{c b}\right|$ and $\Delta m_{d}$ from semi-leptonic $\mathrm{B}^{0}$-decays and $\mathrm{B}^{0}-\overline{\mathrm{B}}^{0}$ oscillations $^{1}$, $\sin 2 \beta=0.695 \pm 0.055$. After one year of nominal operation of $\mathrm{LHC}$ the data sample collected by $\mathrm{LHCb}$ will be sufficient to determine $\beta$ with a similar accuracy as is foreseen by the Bfactories. The Standard Model analysis predicts a value of the CKM angle $\gamma$ of $64.5^{\circ} \pm 7^{\circ}$. The direct measurement of $\gamma$ is one of the main purposes of $\mathrm{LHCb}^{2}$. The high $\mathrm{b} \overline{\mathrm{b}}$ cross section and luminosity will provide an unprece-

Table 2. The expected statistical precision for various CP parameters is listed, as measured with a few specific decay channels. The expected annual event yields are given for the indicated decays and their charge conjugates, together with the estimated $B / S$, or $90 \%$ CL upper limits.

\begin{tabular}{|c|l|c|c|c|}
\hline Quantity & Channel & Yield & $\mathrm{B} / \mathrm{S}$ & Precision \\
\hline \hline $\sin 2 \beta$ & $\mathrm{B}^{0} \rightarrow \mathrm{J} / \psi\left(\mu^{+} \mu^{-}\right) \mathrm{K}_{\mathrm{S}}$ & $216 \mathrm{k}$ & 0.8 & 0.022 \\
$\chi$ & $\mathrm{B}_{\mathrm{S}}^{0} \rightarrow \mathrm{J} / \psi\left(\mu^{+} \mu^{-}\right) \phi$ & $100 \mathrm{k}$ & $<0.3$ & 0.058 \\
\hline$\gamma-2 \chi$ & $\mathrm{B}_{\mathrm{S}}^{0} \rightarrow \mathrm{D}_{\mathrm{S}}^{ \pm} \mathrm{K}^{\mp}$ & $5.4 \mathrm{k}$ & $<1.0$ & $14-15^{\circ}$ \\
$\gamma$ & $\mathrm{B}_{d(s) \rightarrow \pi^{+} \pi^{-}\left(\mathrm{K}^{+} \mathrm{K}^{-}\right)}^{0}$ & $26 \mathrm{k}(37 \mathrm{k})$ & $<0.7(0.31)$ & $4-6^{o}$ \\
$\gamma$ & $\mathrm{B}^{0} \rightarrow \mathrm{D}^{0} \mathrm{~K}^{* 0}$ & $3.4 \mathrm{k}$ & $<0.5$ & $7-8^{o}$ \\
$\Delta m_{s}$ & $\mathrm{~B}_{\mathrm{S}}^{0} \rightarrow \mathrm{D}_{\mathrm{s}}^{-} \pi^{+}$ & $80 \mathrm{k}$ & 0.32 & $0.06 \%$ \\
\hline rare decay & $\mathrm{B}^{0} \rightarrow \mathrm{K}^{* 0} \gamma$ & $35 \mathrm{k}$ & $<0.7$ & \\
\hline
\end{tabular}


dented large sample of $\mathrm{B}_{\mathrm{s}}^{0}$-mesons, with which this can be achieved. In Table 2 three decay channels are listed that will enable the extraction of $\gamma$.

- The decay $\mathrm{B}_{\mathrm{s}}^{0} \rightarrow \mathrm{D}_{\mathrm{s}}^{ \pm} \mathrm{K}^{\mp}$ measures the angle $\gamma-2 \chi$, where the $\mathrm{B}_{\mathrm{s}}^{0}$ mixing phase $\chi$ arises from the $\mathrm{B}_{\mathrm{s}}^{0}-\overline{\mathrm{B}}_{\mathrm{s}}^{0}$ mixing and is obtained from the $\mathrm{B}_{\mathrm{s}}^{0} \rightarrow \mathrm{J} / \psi\left(\mu^{+} \mu^{-}\right) \phi$ analysis. The angle $\gamma$ comes from the phase of $\left|V_{u b}\right|$ in the tree level decay. Both the $\mathrm{B}_{\mathrm{s}}^{0}$ and the $\overline{\mathrm{B}}_{\mathrm{s}}^{0}$ meson can decay into $\mathrm{D}_{\mathrm{s}}^{+} \mathrm{K}^{-}$and $\mathrm{D}_{\mathrm{s}}^{-} \mathrm{K}^{+}$, causing large interference effects and good sensitivity to $\gamma$.

- A comparison of the decays $\mathrm{B}^{0} \rightarrow \pi^{+} \pi^{-}$and $\mathrm{B}_{\mathrm{s}}^{0} \rightarrow \mathrm{K}^{+} \mathrm{K}^{-}$will provide a second theoretically clean measurement of $\gamma$. The method relies only on the assumption of $\mathrm{SU}(3)$ symmetry, reducing largely the uncertainties from penguin contributions. New particles participating in the loop might result in a different value of $\gamma$, compared to the $\mathrm{B}_{\mathrm{s}}^{0} \rightarrow \mathrm{D}_{\mathrm{s}}^{ \pm} \mathrm{K}^{\mp}$-result, hence indicating new physics.

- The decay $\mathrm{B}^{0} \rightarrow \stackrel{\left(-\mathrm{D}^{0}\right.}{ } \mathrm{K}^{* 0}$ is 'self-tagging' by the flavour of $\mathrm{K}^{* 0}$ and therefore no time dependent measurements are required, resulting in small statistical uncertainty. Both $\gamma$ and the strong phase difference $\Delta$ can be obtained by measuring the three time-integrated decays (to $\mathrm{D}^{0}, \overline{\mathrm{D}}^{0}$ and the $\mathrm{CP}$ eigenstate $\mathrm{D}_{\mathrm{CP}}^{0}$ ), and their charge conjugates.

In addition, basic parameters like the mass difference $\Delta m_{s}$ and lifetime difference $\Delta \Gamma_{s}$ of the two $\mathrm{B}_{\mathrm{s}}^{0}$-mass eigenstates will be determined with high precision. Finally, any new particles present in the loop diagrams could significantly enhance the branching ratios of channels with very low branching ratios in the Standard Model, such as $\mathrm{B}^{0} \rightarrow \mathrm{K}^{* 0} \gamma$, or with the experimentally very clean decays containing muons, $\mathrm{B}^{0} \rightarrow \mu^{+} \mu^{-} \mathrm{K}^{* 0}$, $\mathrm{B}_{\mathrm{s}}^{0} \rightarrow \mu^{+} \mu^{-}$and $\mathrm{B}^{0} \rightarrow \mu^{+} \mu^{-}$, with estimated Standard Model branching ratios of $8 \cdot 10^{-7}, 4 \cdot 10^{-9}$ and $10^{-10}$, respectively.

\section{References}

1. M. Battaglia et al., The CKM matrix and the Unitarity Triangle, hep$\mathrm{ph} / 0304132$.

2. The LHCb Collaboration, R. Antunes Nobrega et al., Technical Design Report - Reoptimized Detector, CERN/LHCC 2003-030 (Sep 2003).

3. The LHCb Collaboration, R. Antunes Nobrega et al., Trigger System Technical Design Report, CERN/LHCC 2003-031 (Sep 2003).

4. BABAR Collaboration, B. Aubert et al., Phys.Rev.Lett. 89 (2002) 201802; BELLE Collaboration, K. Abe et al., Phys.Rev. D66 (2002) 071102. 